\title{
Ginsenoside Rg1 Promotes the Migration of Olfactory Ensheathing Cells via the PI3K/Akt Pathway to Repair Rat Spinal Cord Injury
}

\author{
Yin-Yao Tang, ${ }^{a, \#}$ Wei-Xiao Guo, ${ }^{b, \#}$ Zheng-Feng Lu, ${ }^{*, a}$ Mao-Hua Cheng, ${ }^{a}$ Yi-Xin Shen, ${ }^{a}$ and \\ Ying-Zi Zhang ${ }^{a}$ \\ ${ }^{a}$ Department of Orthopaedics, The Second Affiliated Hospital of Soochow University; Suzhou 215000, China: and \\ ${ }^{b}$ Department of Orthopaedics, Suzhou Kowloon Hospital, Shanghai Jiao Tong University School of Medicine; Suzhou \\ 215021, China.
}

Received November 18, 2016; accepted June 30, 2017

\begin{abstract}
The aim of this study was to determine the effects of ginsenoside Rg1 on the migration of olfactory ensheathing cells (OECs) in vitro, and its influence on the therapeutic efficacy of OECs transplanted in vivo for the treatment of spinal cord injury (SCI). Primary cultured and purified OECs (prepared from rats) were treated with ginsenoside Rg1. The wound healing test indicated that ginsenoside Rg1 promoted the migration of OECs. Real-time RT-PCR demonstrated that ginsenoside Rg1 upregulated the expression of migration-related factors of OECs, including matrix metalloproteinases-2 (MMP-2), MMP-9, and neural cell adhesion molecule 1 (NCAM1). Moreover, Western blot analysis indicated that ginsenoside Rg1 significantly promoted the migration of OECs via the phosphatidylinositol 3-kinase (PI3K)/Akt pathway. An SCI rat model was induced in vivo using a revised Allen's method. The Basso, Beattie, and Bresnahan (BBB) scores and histological analysis demonstrated that OECs, which were treated with ginsenoside Rg1, exhibited significant improvement in SCI compared with both the control group and the OEC group. Thus, ginsenoside Rg1 may represent a novel treatment target for SCI.
\end{abstract}

Key words olfactory ensheathing cell; ginsenoside Rg1; migration; spinal cord injury

In an injured spinal cord, it is very difficult to regenerate neurites without a myelin sheath. Cell transplantation has been extensively used to treat spinal cord injury (SCI) in recent years. ${ }^{1,2)}$ Schwann cells, neural stem cells, bone marrow mesenchymal stem cells, and olfactory ensheathing cells (OECs) are frequently used in cell transplantation. In particular, OECs are one of the most promising seed cells. ${ }^{3)}$ They protect neurons by secreting a neurotrophic factor, which inhibits scar formation; they also serve as a bridge for injured neurite growth and promote regeneration of axons. In addition, OECs play a crucial role in neuronal protection because they have the ability to migrate, proliferate, secrete various neurotrophic factors, and myelinate regenerating axons. ${ }^{4)}$ Furthermore, OECs facilitate the in vivo repair of axons, which are damaged in patients with SCI. In the repair of damaged axons, OECs improve the migration and adhesion abilities of cells. ${ }^{5}$ However, previous research indicates that the proliferation ability and biological activity of OECs significantly decreased in an in vitro culture. ${ }^{6}$ ) Therefore, it is critical to improve the neural repairing ability of OECs that are associated with SCI via the enhancement of the biological activity of cells. Recent studies conducted by our team ${ }^{7,8)}$ have demonstrated that silk fibroin nanofibers enhanced the adhesion, growth and migration of OECs in vitro. However, it is difficult to accomplish this strategy in vivo. Thus, the present research was designed to identify additional factors that can improve the migration ability of OECs both in vitro and in vivo, in order to promote the translation from bench to bedside.

Ginsenoside Rg1 is a major bioactive ingredient of Panax ginseng. Panax ginseng has been well-established as a traditional herbal medicine in Asia for more than 2000 years, and

\footnotetext{
\# These authors contributed equally to this work.

has exhibited pharmacological activities that affect the cardiovascular, endocrine, immune and central nervous systems, ${ }^{9}{ }^{9}$ especially in repairing damaged neurons. The wide range of pharmacological actions of ginseng may be a result of the variety of its active ingredients-ginsenosides. ${ }^{10)}$ Ginsenosides, extracted from ginseng root, have been demonstrated to promote peripheral axonal regeneration, ${ }^{11)}$ and the neuroprotective effects of ginseng components in injured spinal cord tissues ${ }^{12}$ ) or ischemic brains have been reported. ${ }^{13)}$ In previous studies, it has been reported that Rg1 protects neurons and promotes cell proliferation and axonal regeneration. ${ }^{11,14)}$ With a structure similar to glucocorticoids, ginsenoside Rg1 also regulates the phenotypic activity of glial cells. Furthermore, Rg1 offers protection to neuronal cells damaged as a result of SCI. ${ }^{12,15,16)}$

Our previous studies have demonstrated that ginsenoside $\operatorname{Rg} 1$ promotes the proliferation of OECs and upregulates the expression and secretion of neurotrophic factors, including brainderived neurotrophic factor (BDNF), glial-derived neurotrophic factor (GDNF), and nerve growth factor (NGF). ${ }^{17)}$ However, it is unclear whether ginsenoside Rg1 can promote OEC migration or facilitate SCI repair. The extent of neuronal protection offered by OECs depends on their migration character, the extent of myelin regeneration is also governed by the migrating ability of OECs. OECs migrate into the adjacent regions of the central nervous system (CNS) to facilitate axonal contact. ${ }^{4}$ Moreover, they also have the ability to myelinate axons under certain pathological conditions. However, following cell transplantation, OECs have limited survival capacity and migration ability. To the best of our knowledge, this is the first study to determine whether ginsenoside Rg1 influences the mechanism governing the migration and other bioactivities of OECs. Furthermore, this study also preliminarily investigated the ability of ginsenoside Rg1 to influence the repair of SCI in vivo. 


\section{MATERIALS AND METHODS}

Cell Culture and Treatments The experimental procedures were approved by the Animal Care and Use Committee of Soochow University, Jiangsu, China. OECs were primarily cultured and purified using simplified methods, as previously described. ${ }^{18)}$ Briefly, 30 five-day-old neonatal Sprague-Dawley rats (male and female; Zhaoyan Co., Ltd., Suzhou, China) were anesthetized via an intraperitoneal injection of $2 \%$ pentobarbital sodium salt (TIANDZ, Beijing, China) $(30 \mathrm{mg} / \mathrm{kg})$ and decapitated. The olfactory bulbs were detached, and the pia mater and capillaries on the surface were subsequently peeled to expose the white matter. The white matter that was present inside the olfactory bulb was subsequently removed. The gray matter of the olfactory bulb was placed in complete Dulbecco's modified Eagle's medium (the DMEM/F-12 medium contained 10\% fetal bovine serum; Hyclone, Logan, UT, U.S.A.). The samples were rapidly sheared using ophthalmic scissors for five minutes and gently blown approximately 15-20 times using a pipette. The homogeneous suspension was directly seeded onto $25-\mathrm{cm}$ culture bottles, which were coated with poly-L-lysine (PLL; Sigma, St. Louis, MO, U.S.A.). The culture was incubated at $37^{\circ} \mathrm{C}$ in $5 \% \mathrm{CO}_{2}$, and the culture medium was replenished every three days. Following $14 \mathrm{~d}$ of incubation, cells were purified using cytosine arabinoside at different adherent velocities. The purified OECs were identified via flow cytometry using nerve growth factor receptor p75 (NGFRp75) (rabbit polyclonal antibody, sc-6188-R, 1:400; Santa Cruz, San Antonio, TX, U.S.A.). OECs that were added with NGFRp 75 antibody were incubated at $4{ }^{\circ} \mathrm{C}$ for $1.5 \mathrm{~h}$. Subsequently, cells were washed twice with phosphate buffered saline (PBS) and incubated at $4^{\circ} \mathrm{C}$ for $30 \mathrm{~min}$ with an fluorescein isothiocyanate (FITC)-labeled goat anti rabbit immunoglobulin G (IgG) (A0562, Beyotime, Shanghai, China). Then, cells were washed twice with PBS and subsequently analyzed by an FACS Calibur (BD Bioscience, San Jose, CA, U.S.A.). The OECs were subsequently divided into two groups: the experimental and control groups. The experimental group was treated with $40 \mu \mathrm{g} / \mathrm{mL}$ Rg1 solution (Jin Sui Biological Technology Co., Ltd., Shanghai, China), whereas the control group was treated with an equal volume of DMEM/F12 medium. The Rg1 dosage represented the optimal concentration and intervention time as demonstrated by our previous study. ${ }^{17)}$

Wound Healing Test Cells that were included in the experimental and control groups were initially seeded in 6-well plates (coated with PLL). ${ }^{18)}$ The surfaces of the plates were scratched using a $200 \mu \mathrm{L}$ sterile pipette tip to induce an artificial "wound" (approximately $1.0 \mathrm{~mm}$ gap) at $0 \mathrm{~h}$. The floating cell debris was removed by washing twice with PBS. Then, the cells were placed in a complete medium. Cells were randomly selected from the area bordering the initial wound. The cell numbers that had advanced into the cell-free space were counted at $0,4,8,12$, and $24 \mathrm{~h}$. Photographs were taken using an inverted Olympus microscope (Olympus, Tokyo, Japan). Cell migration was determined using Image-Pro Plus software (Media Cybernetics, Bethesda, MD, U.S.A.).

Transwell Purified OECs were divided into four groups: the Rg1 group, control group, and two inhibition groups. In the Rg1 group, the purified OECs were treated with Rg1 for $72 \mathrm{~h}$ at an optimum dosage of $40 \mu \mathrm{g} / \mathrm{mL}$. In the control group, the purified OECs received an equal volume of DMEM/F12 medium. In one inhibition group, the purified OECs were pretreated with LY294002 (Cell Signaling Technology, Danvers, MA, U.S.A.) $(20 \mu \mathrm{M}$, a specific phosphatidylinositol-3-kinase, $\mathrm{PI} 3 \mathrm{~K}$, inhibitor) at $37^{\circ} \mathrm{C}$ for $2 \mathrm{~h}$ prior to the addition of $\mathrm{Rg} 1$, whereas the other inhibition group was treated with LY294002 only. The migration assays were performed using transwell chambers with an $8-\mu \mathrm{m}$ pore size (Corning Costar, Lowell, MA, U.S.A.) ${ }^{19)}$ Cell suspensions $\left(200 \mu \mathrm{L}, 2 \times 10^{5}\right.$ in medium) were added to the upper chamber. The lower chambers were filled with $600 \mu \mathrm{L}$ of complete medium. Then, the plates were incubated at $37^{\circ} \mathrm{C}$ for $24 \mathrm{~h}$. Following incubation, the remaining cells in the upper surface of the filter were removed using a cotton swab. The migrated cells on the lower surface of the filter membrane were stained with crystal violet and subsequently counted under a light microscope (Olympus, Tokyo, Japan). The number of cells was counted in 10 separate high power fields $(\times 200)$.

Real-Time RT-PCR The total RNA of the cells in the Rg1 treated and control groups was isolated using Trizol reagent and then reverse transcribed into cDNA according to the manufacturer's instructions of the TaKaRa kit (TaKaRa Biotechnology, Shiga, Japan). RT-PCR was performed using the QuantiTect SYBR Green RT-PCR Kit (Thermo Scientific, Waltham, MA, U.S.A.). The PCR protocol conditions were as follows: $95^{\circ} \mathrm{C}$ for $10 \mathrm{~min}$, followed by 40 cycles at various temperatures/times $\left(95^{\circ} \mathrm{C}\right.$ for $20 \mathrm{~s}, 60^{\circ}$ for $20 \mathrm{~s}$, and $72^{\circ} \mathrm{C}$ for $20 \mathrm{~s}$ ). A melting curve analysis was conducted at the end of the amplification period. The gene changes in the two groups were calculated by normalizing the $\mathrm{Ct}$ values to $\beta$-actin. The primer sequences were as follows: matrix metalloproteinases-2 (MMP-2), 5'-GAA ATTCAGAAGGTGCCCCA-3' (forward), 5'-AGG TGT AGA TAGGGGCCA TC-3' (reverse); MMP-9, 5'GCA TCT GTA TGG TCG TGGCT-3' (forward), 5'-CTGTAG GGGCCTCAGAAGGA-3' (reverse); neural cell adhesion molecule 1 (NCAM1), 5'-CGACCAGAGAAGCAAGAGAC-3' (forward), 5'-CTC GGA ACC AGG AGA TTG TG-3' (reverse); $\beta$-actin, 5'-CAC CCGCGA GTA CAACCT TC-3' (forward), 5'-CCC ATA CCC ACC ATC ACA CC-3' (reverse).

The primers were designed using Primer Premier 5 software, checked for self-assembly amplification, and synthesized by Sangon Biotech (Shanghai, China).

Western Blotting To determine the effect of ginsenoside Rg1 on Akt phosphorylation, the Rg1 group, control group, and two inhibition group cells were lysed, and the protein from the cytoplasm was extracted according to the kit instructions (Beyotime, Shanghai, China). The protein concentrations were subsequently determined using a BCA kit (Beyotime, Shanghai, China). The proteins were separated via sodium dodecyl sulfonate-polyacrylamide gel electrophoresis (SDSPAGE) and transferred to a polyvinylidene difluoride (PVDF) membrane. The membrane was blocked with a solution that contained 5\% nonfat dry milk and PBS buffer overnight at $4^{\circ} \mathrm{C}$. After the non-specific binding was blocked, the membrane was incubated with primary antibodies (PI3K, rabbit polyclonal, \#4292, phosphorylated Akt-1, rabbit monoclonal, D7F10, total Akt-1, rabbit monoclonal, $\mathrm{C} 73 \mathrm{H} 10$, and $\beta$-actin, rabbit polyclonal, \#4967, 1:1000; Cell Signaling Technology, Danvers, MA, U.S.A.) for $2.5 \mathrm{~h}$ at room temperature. The membrane was then incubated with secondary antibodies that were conjugated with horseradish peroxidase (goat anti rabbit IgG, 1:3000; Cell Signaling Technology) for $1.5 \mathrm{~h}$ at room 
temperature. The immunoreactive bands were visualized using the enhanced chemiluminescent (ECL) detection kit that exposed the film. The Western blot band densitometry analysis was performed, and the data were analyzed using the Image J program.

Establishment of SCI Rat Model and Cell Transplantation The SCI model was established using a modified version of Allen's weight dropping method. ${ }^{19)}$ Briefly, 30 adult Sprague-Dawley rats (Zhaoyan Co., Ltd., Suzhou, China) were anesthetized via an intraperitoneal injection of $5 \%$ chloral hydrate $(0.7 \mathrm{~mL} / 100 \mathrm{~g}$; BBI Life Sciences Corporation, Shanghai, China) and then fixed on an operative board. The skin was disinfected, and a laminectomy was performed at T9-T10 level to fully expose the spinal cord. An Allen's hit was used to establish a rat injury model. In this revised model, a Kirschner wire that weighed $10 \mathrm{~g}$ was inserted into a tube and dropped from a height of $4 \mathrm{~cm}$, hitting the exposed spinal cord. The rats were then randomized to receive the following modes of treatment for SCI: OEC treatment (OEC group, $n=10)$, OEC and Rg1 treatment (Rg1+OEC group, OECs treated with Rg1 at a concentration of $40 \mu \mathrm{g} / \mathrm{mL}$ for $72 \mathrm{~h}$ prior to the surgery, $n=10$ ), or DMEM/F12 treatment as the control group (SCI group, $n=10$ ). Five microliter of OECs at a concentration of $5 \times 10^{7} / \mathrm{mL}$ were injected into the spinal cord around the injury site using a microinjector (Anting Co., Ltd., Shanghai, China). Following the injection, the muscles and skin were sutured along the midline of the injury site. All rats who underwent the operation were administered penicillin ( $4 \mathrm{U} / \mathrm{mL}, 0.5 \mathrm{~mL} / \mathrm{d})$ for three days.

Behavioral Assessment Functional recovery was scored according to the Basso, Beattie, and Bresnahan (BBB) scale ${ }^{20)}$ on days $1,3,7,14,21$, and 28 postoperatively. Locomotor activity was evaluated using the open-field walking scoring system and was measured for $5 \mathrm{~min}$. One animal at a time was allowed to move freely inside a circular plastic tray $(90 \mathrm{~cm}$ diameter $\times 24 \mathrm{~cm}$ wall height). Two independent examiners observed the hindlimb movements of the rat and scored the locomotor function according to the $\mathrm{BBB}$ scale, which ranges from 0 (no hindlimb movement) to 21 (normal movementcoordinated gait). The final score of each animal represented the mean value of both examiners.

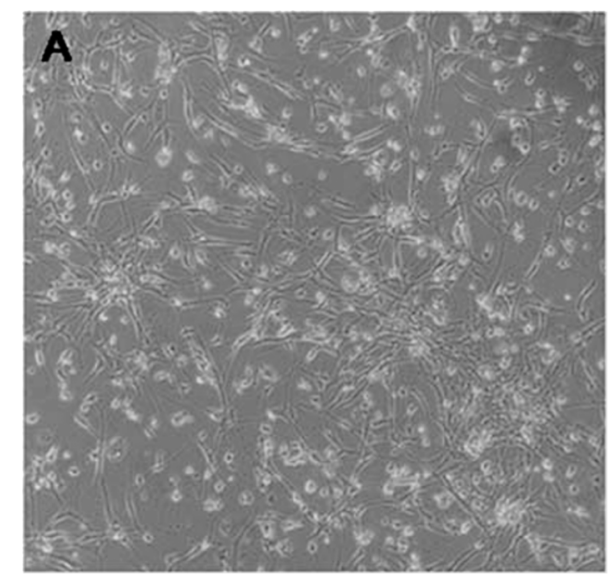

Immunohistochemistry Twenty-eight days after the operation, the rats were anesthetized with an intraperitoneal injection of chloral hydrate $(0.7 \mathrm{~mL} / 100 \mathrm{~g})$ and perfused through the left ventricle with $4 \%$ formaldehyde solution to fix the spinal cord. The tissues were then placed in paraffin. The spinal cord tissue of each rat was divided into three sections, which were subsequently stained with hematoxylin-eosin (HE), glial fibrillary acidic protein (GFAP), and neuron specific enolase (NSE), respectively. The samples were treated with primary antibodies (anti-GFAP antibody: rabbit polyclonal, $\mathrm{CP}$ 040 A, B; Biocare Medical, Concord, CA, U.S.A., Anti-NSE antibody:rabbit polyclonal, ab834; Abcam, Cambridge, MA, U.S.A.) at a concentration of $1: 500$ overnight at $4{ }^{\circ} \mathrm{C}$, washed three times with PBS, and incubated with secondary antibodies (goat anti rabbit $\mathrm{IgG}$ ) for $30 \mathrm{~min}$ at $37^{\circ} \mathrm{C}$. Thereafter, they were washed again with PBS, treated with diaminobenzidine (DAB) in hydrogen peroxide, and mounted with neutral gum. Images were observed under an upright microscope. The data were analyzed using Image-Pro Plus software (Media Cybernetics, U.S.A.).

Statistical Analyses The results are presented in terms of the mean \pm standard deviation (S.D.). Statistically significant differences between data (defined as $p<0.05$ ) were evaluated by two-sided Student's $t$-test or one-way ANOVA followed by least significant difference (LSD) post hoc test (SPSS version 17.0, SPSS Inc., Chicago, IL, U.S.A.). All experiments were performed in quadruplicate.

\section{RESULTS}

Purification and Characterization of OECs Following the three-step purification procedure, there were numerous bipolar and tripolar cells at the bottom of the flask (Fig. 1A). OECs were specifically labeled using the NGFRp75 antibody. Up to $97.7 \%$ positive cells were identified (Fig. 1B). The cell purity met the requirements of the subsequent experiments.

Migration-Enhancing Effects of Ginsenoside Rg1 on OECs We evaluated the migration effect of ginsenoside Rg1 on OECs using the wound healing test. Images were taken at $0,4,8,12$, and $24 \mathrm{~h}$. A greater number of cells passed through the scratches in the ginsenoside $\mathrm{Rg} 1$ treated group within

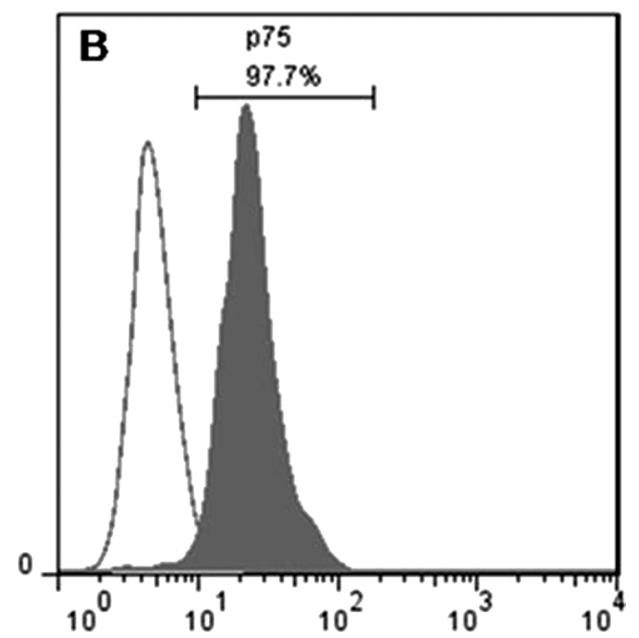

Fig. 1. OEC Morphology and Identification

High-purity olfactory ensheathing cells (OECs) were maintained, following a three-step purification process (A, $\times 200)$. The expression of p75 on the cell surface was determined via flow cytometry. Purified OECs were $97.7 \%$ positive for $\mathrm{p} 75$ (B). 

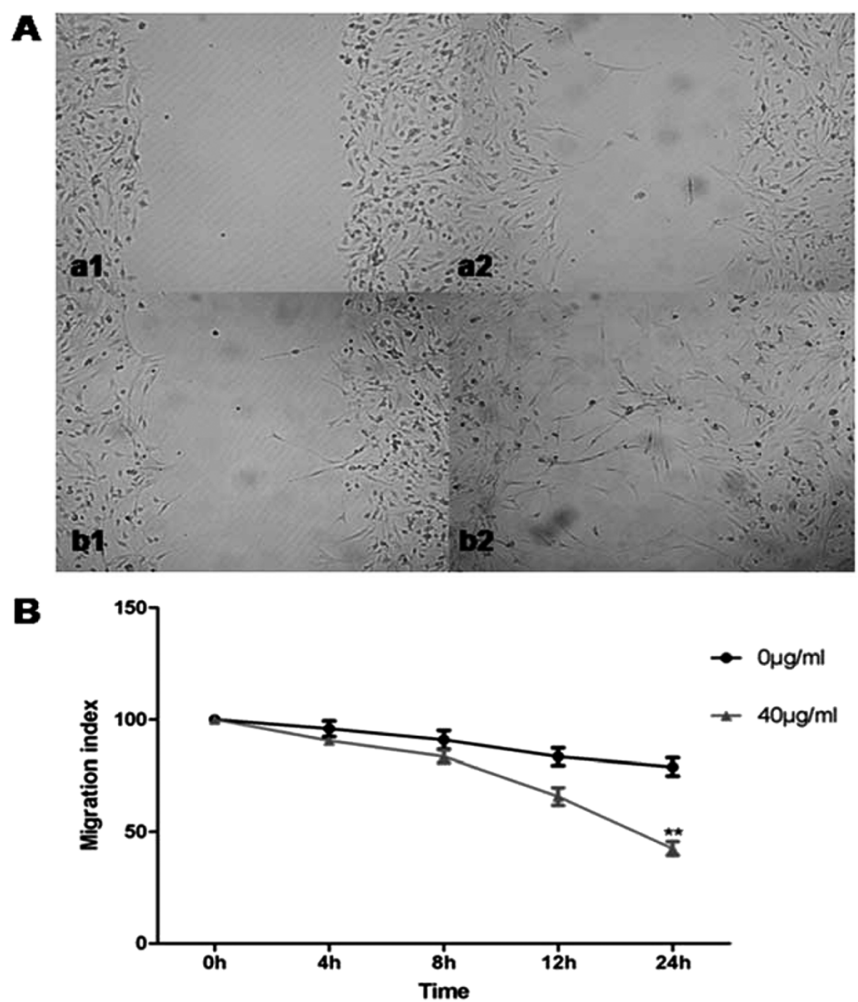

Fig. 2. Wound Healing Test

A: Cell monolayers were wounded by scraping at $0 \mathrm{~h}$ (a1: control group; b1: $\mathrm{Rg} 1$ group; $\times 200$ ). After $24 \mathrm{~h}$ of culture, more cells had migrated into the scratches in the Rg1 group (b2) compared with the control group (a2). B: The wound healing test results indicated that $\mathrm{Rg} 1$ promotes the migration of OECs into the scratches at $24 \mathrm{~h}\left(F_{1,8}=269.2, * * p<0.01, N=5\right)$. The migration index was expressed as a value of the percentage of blank area (uncovered by the cell monolayer) and accounted for the original scratch area. Error bars represent the standard differentiation of four duplicates. $p$ Values are based on one-way ANOVA. 24h (Fig. 2A). Data analysis across the different time points indicated that ginsenoside $\mathrm{Rg} 1$ promoted OEC migration. Compared with the control group, the scratch area (the reduction in the scratch area was determined every $4 \mathrm{~h}$ ) was reduced at a faster rate in the Rg1+OEC group, especially at $24 \mathrm{~h}$ (Fig. 2B). This finding indicated that ginsenoside Rg1 promoted the migration effect of OECs.

Ginsenoside Rg1 Upregulated the Expression of Migration-Related Factors in OECs The expression of MMPs and NCAM1 is crucial in determining the migration ability of OECs. Thus, it is necessary to determine whether MMPs and NCAM1 are involved in the promotion of migration by ginsenoside Rg1. The effects of ginsenoside Rg1 on MMPs and NCAM1 were investigated using RT-PCR. As shown in Fig. 3, ginsenoside Rg1 upregulated the expression of MMP-2 $(p<0.05)$, MMP-9 $(p<0.01)$, and NCAM1 $(p<0.01)$. These findings indicated that ginsenoside Rg1 affected the expression of genes that influence the migration ability of OECs.

Ginsenoside Rg1 Enhanced OEC Migration via the

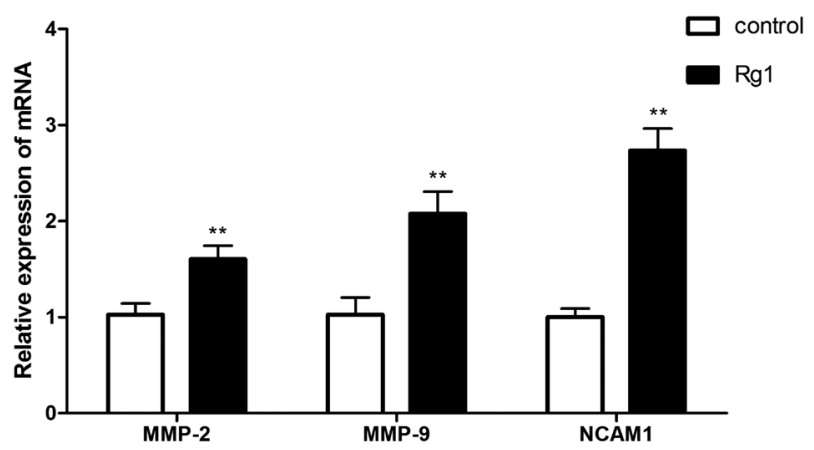

Fig. 3. mRNA Expressions of Migration-Related Factors

Real-time PCR results indicated that the expression levels of MMP-2, MMP-9, and NCAM1 mRNA were significantly increased in the $\operatorname{Rg} 1$ group $(* * p<0.01)$. $p$ Values are based on independent-samples $t$-test $(n=3)$.

\section{A}
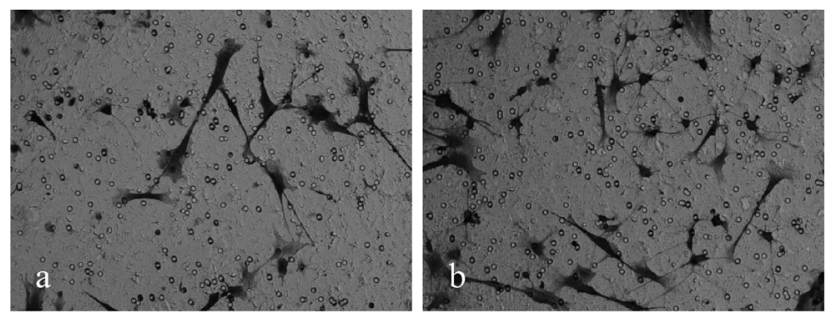

B

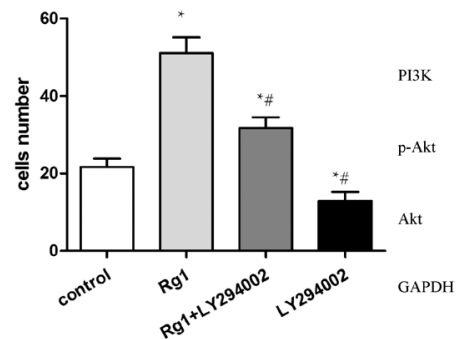

$\mathrm{C}$

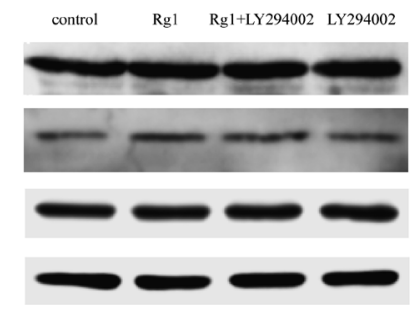

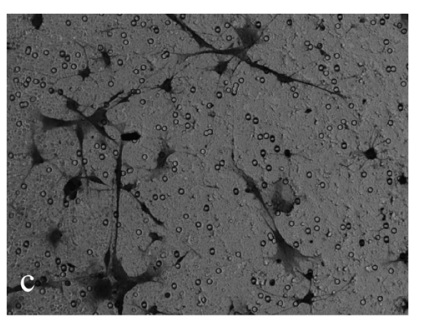

$\mathrm{D}$

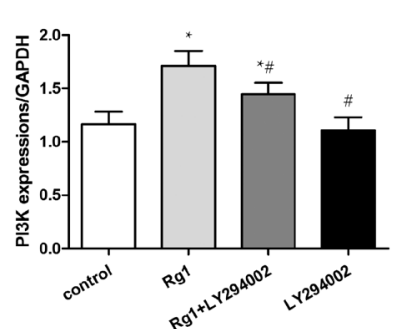

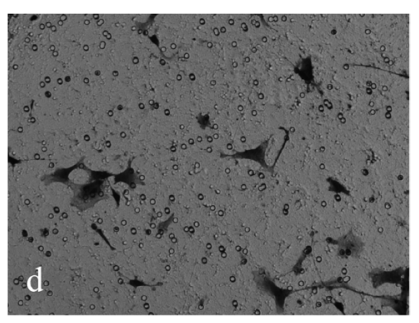

E

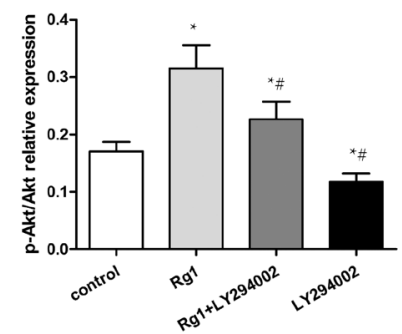

Fig. 4. Transwell Chamber Experiments and Western Blotting

The transwell results indicated that (A, ×200) cell migration was increased after ginsenoside Rg1 treatment (b) compared with the control group (a). LY294002 (a specific PI3K inhibitor) significantly decreased the effects of Rg1 on OECs (c). LY294002 markedly suppressed the migration of OECs (d). Migrated cells were counted in ten individual microscopic fields and analyzed via ANOVA (B, $F_{3,36}=9.643,{ }^{*} p<0.05, n=10$ ). Western blot analysis indicated increased activation (phosphorylation) of Akt following Rg1 stimulation in OECs (C). $p$ Values are based on one-way ANOVA. Multiple comparison between the groups was performed using the LSD method $(*$ indicates a significant difference compared with the sham control, $p<0.05$; \# indicates a significant difference compared with the $\operatorname{Rg} 1$ group, $p<0.05$ ). 
PI3K/AKT Signaling Pathway To further determine the molecular mechanism through which ginsenoside Rg1 affected the migration ability of OECs, the transwell test and Western blotting analysis were conducted. The results of the transwell test indicated that the number of migrated OECs in the lower chamber was significantly upregulated by ginsenoside Rg1 $(p<0.05)$. However, this promoting effect was markedly suppressed by LY294002 (Figs. 4A, B). Compared with the control group, the PI3K protein and the p-Akt/Akt relative expression were significantly increased following ginsenoside Rg1 treatment (Figs. 4D, E* $*<0.05$ ); however, the total-Akt protein levels were not affected by ginsenoside Rg1 (Fig. 4C). Compared with the Rg1 group, the PI3K protein and the p-Akt/Akt relative expression were significantly decreased in the two inhibition groups (Figs. 4D, E $\# p<0.05$ ). In summary, these data indicated that ginsenoside Rg1 promoted OEC migration by enhancing the activation of the PI3K/Akt signaling pathway.

Locomotor Function Recovery and Histological Results Locomotor function was measured using the BBB scale. The BBB scores significantly decreased $(p<0.05)$ immediately after SCI (all animals survived injury model induction). The animals recovered gradually within four weeks. In the first 3 weeks, the BBB scores did not differ between the three groups. From day 21, the BBB scores were significantly increased in the Rg1+OEC group compared with the OEC and

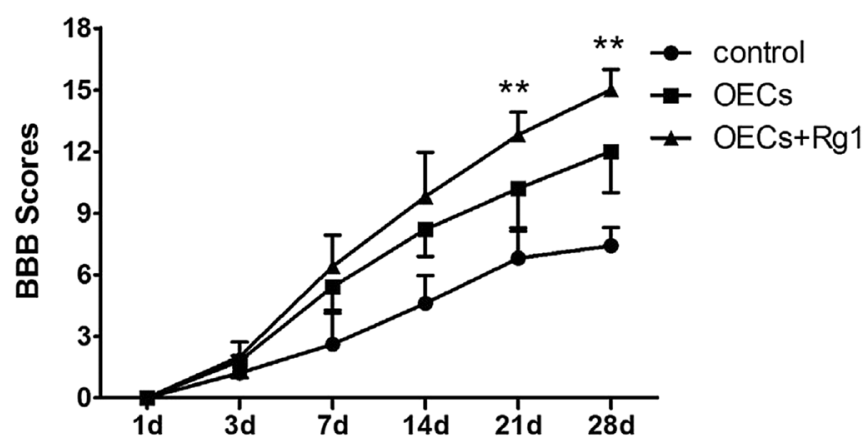

Fig. 5. BBB Scores of the SCI Model

The locomotor recovery curves of a representative rat model indicated that $\mathrm{Rg} 1$ significantly promoted the functional recovery of OECs in SCI $\left(21 \mathrm{~d}: F_{2,12}=20.576\right.$ $\left.* * p<0.01 ; 28 \mathrm{~d}: F_{2,12}=37.897, * * p<0.01\right)$. LSD test following ANOVA $(n=5)$.

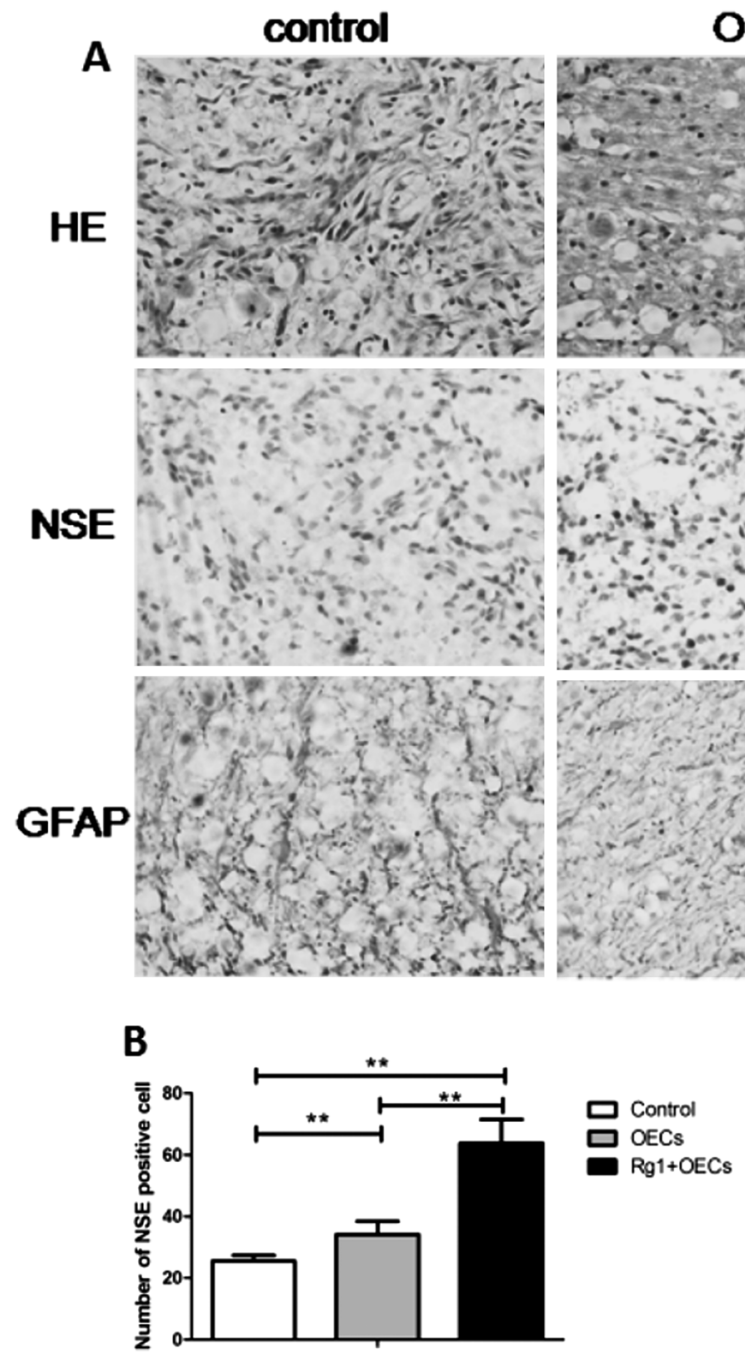

OECS
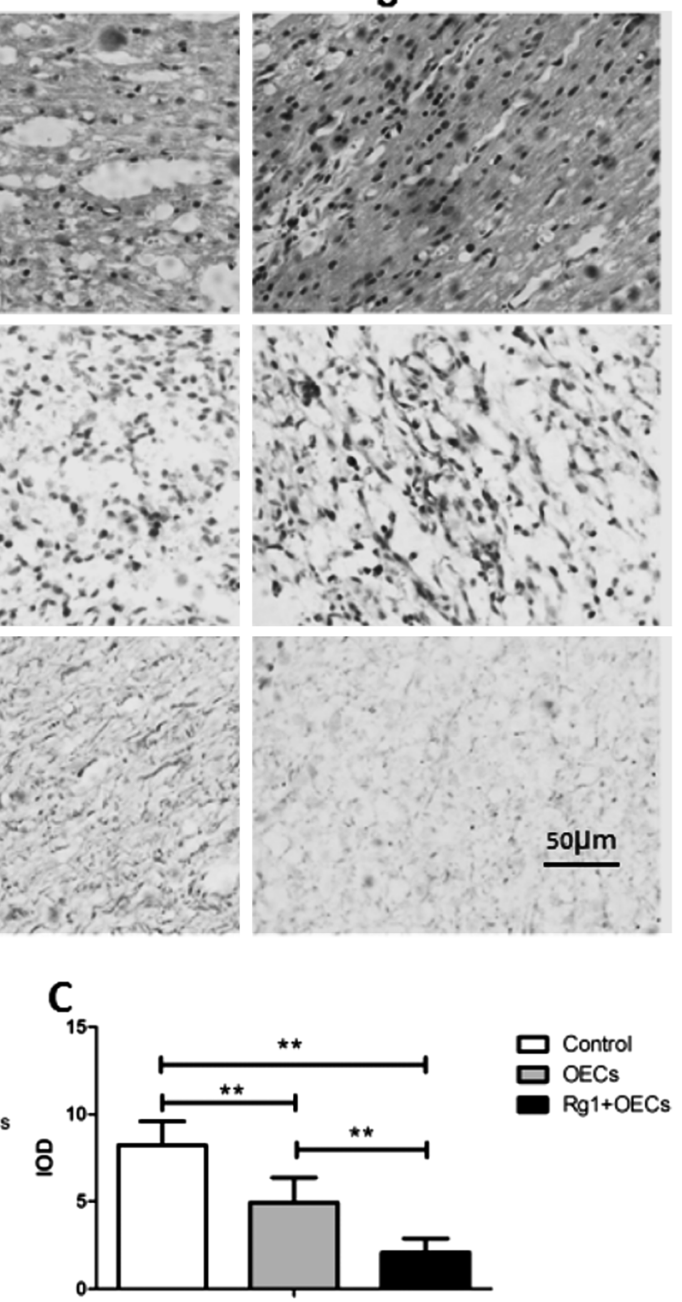

Fig. 6. Immunohistochemistry of the SCI Model

HE stains indicated less damage in the Rg1+OEC group compared with the OEC and control groups. The NSE positive cell number was larger in the Rg1+OEC group compared with the other two groups $\left(\mathrm{B}, F_{2,15}=90.211, * * p<0.01\right)$. The mean IOD of GFAP in the Rg1+OEC group was significantly lower than the OEC and control groups $\left(\mathrm{C}, F_{2,15}=18.101, * * p<0.01\right)$. B and $\mathrm{C}$ indicated that $\mathrm{Rg} 1$ promoted the positive effects of OECs transplanted in SCI via increased neuronal survival and less tissue damage (LSD test following ANOVA, $n=6$ ). 
control groups (Fig. 5).

The histological analysis indicated that there were more delicate changes in the recovery of tissues treated with transplanted OECs subjected to ginsenoside Rg1 treatment. The HE stains demonstrated that there were less cell necrosis and vacuolization in the Rg1+OEC group compared with the OEC and control groups. A clear cell outline and neat tissue arrangement were present in the Rg1+OEC group (Fig. 6A).

We subsequently evaluated nerve cell survival via NSE stains. Surviving neurons were stained yellow. Figure 6A indicates that the $\mathrm{Rg} 1+\mathrm{OEC}$ group exhibited a significantly increased number of NSE positive cells, followed by the OEC group. There were very few NSE positive cells in the control group $(p<0.05$; Fig. 6B). Based on these results, the proliferation of astrocytes that occurred after SCI was specifically determined by GFAP staining. As shown in Fig. 6A, after OECs were transplanted into the injured spinal cord, astrocyte proliferation was inhibited. This inhibitory effect was more obvious in the Rg1+OEC group. Furthermore, there were significant differences in the integrated optical density (IOD) value of GFAP among the three groups (Fig. 6C).

\section{DISCUSSION}

OEC transplantation has emerged as a promising therapeutic strategy to repair SCI because OECs promote neurite regeneration and restore functional recovery following SCI. The migration of OECs is essential for neural regeneration. ${ }^{21)}$ However, inhibitory molecules were persistently present in the spinal cord lesion, which inhibited OEC migration. ${ }^{22)}$ Thus, it is urgently necessary to improve the migration ability of OECs. The aim of our experiments was to develop a feasible and effective method of improving OEC migration. As previously reported, ginsenoside Rg1 enhanced the migration of Schwann cells cultivated in vitro. ${ }^{23)}$ Furthermore, OECs exhibited similar characteristics as Schwann cells. Therefore, we hypothesized that ginsenoside Rg1 can also improve the migratory ability of OECs.

In this study, the wound healing test was utilized to investigate OEC migration. The findings indicate that in the ginsenoside Rg1 group, more cells passed through the artificial scratches, which suggests that ginsenoside $\operatorname{Rg} 1$ facilitated OEC migration. It was also determined that the migratory ability of OECs was governed by the expression of migrationrelated factors, such as MMPs and NCAM1. MMP-2 and MMP-9 play important roles in the migration and invasion of various cells, ${ }^{24)}$ including OECs. ${ }^{25,26)}$ Secreted MMPs regulate the activity of the membrane, which is important for both cell migration and axonal regeneration. Furthermore, the downregulation of MMP-2 and MMP-9 also suppresses cell migration. ${ }^{25-27)}$ NCAM1, which is a major component of OEC-induced corticospinal axon elongation, plays a key role in OEC migration. ${ }^{2829)}$ The RT-PCR findings indicate that ginsenoside Rg1 increased the expression of MMP-2, MMP-9, and NCAM1 genes, which stimulate ginsenoside Rg1 in enhancing the migration characteristic of OECs. Thus, these current findings confirm our hypothesis that ginsenoside Rg1 promotes OEC migration and facilitates the repair of SCI via the inhibition of scar formation. Moreover, it establishes a platform that promotes the recovery of injured neurites.

The PI3K/Akt signaling pathway is involved in many cellular processes, including proliferation, apoptosis, cell migration, angiogenesis, invasion, and metastasis. ${ }^{4,30,31)}$ Furthermore, previous studies have demonstrated that BDNF promotes cell proliferation and migration through the PI3K/Akt pathway. ${ }^{32)}$ Moreover, insulin receptor substrate-4 (IRS-4) enhances cell migration via the PI3K/Akt pathway. ${ }^{33)}$ The PI3K/Akt signaling pathway also regulates the expression of MMP-2 and MMP-9. ${ }^{34)}$ Thus, we assumed that ginsenoside Rg1 enhances the migration of OECs via the PI3K/Akt pathway. In order to verify our assumption, we used the PI3K inhibitor LY294002 in the Transwell test. The Western blotting results indicate that ginsenoside Rg1 activates the PI3K/Akt pathway. However, LY294002 blocks the migratory character of OECs, which is promoted by ginsenoside Rg1. These findings suggest that ginsenoside Rg1 promotes OEC migration by activating the PI3K/Akt signaling pathway.

Increasing evidence indicates that ginsenosides help repair damaged neurons, and the extracts of ginsenosides have been demonstrated to promote peripheral axonal regeneration and protect remnant neurons in injured spinal cord tissues. ${ }^{11,12,14,35)}$ We further investigated how ginsenoside Rg1 improved the efficacy of OECs, which were used in the treatment of SCI. An increased frequency of NSE appearance decreased with neuronal injury. ${ }^{36)}$ As demonstrated in our experiment, NSE positive cells, which represented neuronal survival, were significantly increased in the Rg1 interfered OEC group. In addition, ginsenoside Rg1 markedly reduced GFAP expression in the injured spinal cord, which was closely related to the level of damage and scar formation. ${ }^{37)}$

The present study has confirmed that ginsenoside Rg1 enhances the biological activities of OECs by promoting their migration through the PI3K/Akt pathway. Moreover, ginsenoside Rg1 upregulates the expression of factors related to the migration of OECs. Finally, ginsenoside Rg1 enhances the therapeutic effect of OECs in SCI.

Our previous research demonstrated that ginsenoside $\mathrm{Rg} 1$ promotes the proliferation and viability of OECs. Moreover, it significantly increases the expression of mRNA and stimulates the secretion of proteins, GDNF, BDNF, and NGF. ${ }^{12)}$ Furthermore, Rg1 also significantly upregulates the mRNA expression of ciliary neurotrophic factor (CNTF) and vascular endothelial growth factor (VEGF), which play important roles in the repair of central nerves associated with SCI (this finding has been demonstrated in our lab and will be published in another manuscript). In summary, ginsenoside Rg1 not only promotes the proliferation and migration of OECs but it also up-regulates the mRNA expression and protein secretion of neurotrophic factors (GDNF, BDNF, NGF, CNTF, and VEGF) and migration-related factors (MMP-2, MMP-9, and NCAM1). Thus, the curative effect of transplanted OECs is enhanced in SCI.

Nevertheless, there are several limitations that must be discussed in the interpretation of these findings. We could not clearly elucidate the role of OECs, which were treated with ginsenoside Rg1, in the prognosis of SCI in vivo. Moreover, we could not elucidate the in-depth mechanism through which ginsenoside Rg1 improved the biological activity of OECs. In our future research, fluorescent labeled OECs will be used to decipher how OECs act after transplantation in vivo. Nevertheless, these novel studies have confirmed that ginsenoside Rg1 is a safe and efficacious agent that comprehensively improves 
the biological activity of OECs. Thus, ginsenoside Rg1 is a promising therapeutic target in the repair of SCI.

Acknowledgments This study was supported by the National Natural Science Foundation of China (81330026, 30870642), Jiangsu Province Natural Science Foundation (BK20141186).

Conflict of Interest The authors declare no conflict of interest.

\section{REFERENCES}

1) Li J, Lepski G. Cell transplantation for spinal cord injury: a systematic review. Biomed. Res. Int., 2013, 786475 (2013).

2) Fehlings MG, Vawda R. Cellular treatments for spinal cord injury: the time is right for clinical trials. Neurotherapeutics, 8, 704-720 (2011)

3) Raisman G. Repair of spinal cord injury by transplantation of olfactory ensheathing cells. C. R. Biol., 330, 557-560 (2007).

4) Ekberg JA, Amaya D, Mackay-Sim A, St John JA. The migration of olfactory ensheathing cells during development and regeneration. Neurosignals, 20, 147-158 (2012).

5) Pearse DD, Sanchez AR, Pereira FC, Andrade CM, Puzis R, Pressman Y, Golden K, Kitay BM, Blits B, Wood PM, Bunge MB. Transplantation of Schwann cells and/or olfactory ensheathing glia into the contused spinal cord: Survival, migration, axon association, and functional recovery. Glia, 55, 976-1000 (2007)

6) Higginson JR, Barnett SC. The culture of olfactory ensheathing cells (OECs)-A distinct glial cell type. Exp. Neurol., 229, 2-9 (2011).

7) Shen Y, Qian Y, Zhang H, Zuo B, Lu Z, Fan Z, Zhang P, Zhang F, Zhou C. Guidance of olfactory ensheathing cell growth and migration on electrospun silk fibroin scaffolds. Cell Transplant., 19, 147-157 (2010)

8) Fan Z, Shen Y, Zhang F, Zuo B, Lu Q, Wu P, Xie Z, Dong Q, Zhang $\mathrm{H}$. Control of olfactory ensheathing cell behaviors by electrospun silk fibroin fibers. Cell Transplant., 22 (Suppl. 1), S39-S50 (2013).

9) Kitagawa S, Takahashi T, Nabekura T, Tachikawa E, Hasegawa H. Inhibitory effects of ginsenosides and their hydrolyzed metabolites on daunorubicin transport in KB-C2 cells. Biol. Pharm. Bull., 30, 1979-1981 (2007).

10) Wang YZ, Chen J, Chu SF, Wang YS, Wang XY, Chen NH, Zhang JT. Improvement of memory in mice and increase of hippocampal excitability in rats by ginsenoside Rgl's metabolites ginsenoside Rh1 and protopanaxatriol. J. Pharmacol. Sci., 109, 504-510 (2009).

11) Ma J, Li W, Tian R, Lei W. Ginsenoside Rg1 promotes peripheral nerve regeneration in rat model of nerve crush injury. Neurosci. Lett., 478, 66-71 (2010).

12) Liao B, Newmark H, Zhou R. Neuroprotective effects of ginseng total saponin and ginsenosides $\mathrm{Rb} 1$ and $\mathrm{Rg} 1$ on spinal cord neurons in vitro. Exp. Neurol., 173, 224-234 (2002).

13) Sun C, Lai X, Huang X, Zeng Y. Protective effects of ginsenoside Rg1 on astrocytes and cerebral ischemic-reperfusion mice. Biol. Pharm. Bull., 37, 1891-1898 (2014).

14) Wang B, He L, Cui B, Lv H. Protection of ginsenoside Rg1 on central nerve cell damage and the influence on neuron apoptosis. Pak. J. Pharm. Sci., 27 (Suppl.), 2035-2040 (2014).

15) Shen L, Zhang J. Ginsenoside $\operatorname{Rg} 1$ increases ischemia-induced cell proliferation and survival in the dentate gyrus of adult gerbils. $\mathrm{Neu}$ rosci. Lett., 344, 1-4 (2003).

16) Radad K, Gille G, Moldzio R, Saito H, Ishige K, Rausch WD. Ginsenosides Rb1 and Rg1 effects on survival and neurite growth of MPP+-affected mesencephalic dopaminergic cells. J. Neural Transm., 111, 37-45 (2004).
17) Lu ZF, Shen YX, Zhang P, Xu YJ, Fan ZH, Cheng MH, Dong QR. Ginsenoside Rg1 promotes proliferation and neurotrophin expression of olfactory ensheathing cells. J. Asian Nat. Prod. Res., 12, 265-272 (2010)

18) Lu ZF, Shen YX, Zhang P, Fan ZH, Dong QR, Wang M. Simplified methods to isolate culture and purify olfactory ensheathing cells. Neural. Regen. Res., 5, 1495-1499 (2010).

19) Vijayaprakash KM, Sridharan N. An experimental spinal cord injury rat model using customized impact device: a cost-effective approach. J. Pharmacol. Pharmacother., 4, 211-213 (2013).

20) Basso DM, Beattie MS, Bresnahan JC, Anderson DK, Faden AI, Gruner JA, Holford TR, Hsu CY, Noble LJ, Nockels R, Perot PL, Salzman SK, Young W. MASCIS evaluation of open field locomotor scores: effects of experience and teamwork on reliability. Multicenter Animal Spinal Cord Injury Study. J. Neurotrauma, 13, 343-359 (1996)

21) Wang Y, Teng HL, Gao Y, Zhang F, Ding YQ, Huang ZH. Brainderived neurotrophic factor promotes the migration of olfactory ensheathing cells through TRPC channels. Glia, 64, 2154-2165 (2016).

22) Reginensi D, Carulla P, Nocentini S, Seira O, Serra-Picamal $X$, Torres-Espin A, Matamoros-Angles A, Gavin R, Moreno-Flores MT, Wandosell F, Samitier J, Trepat X, Navarro X, del Rio JA. Increased migration of olfactory ensheathing cells secreting the Nogo receptor ectodomain over inhibitory substrates and lesioned spinal cord. Cell. Mol. Life Sci., 72, 2719-2737 (2015).

23) Lu MC, Lai TY, Hwang JM, Chen HT, Chang SH, Tsai FJ, Wang HL, Lin CC, Kuo WW, Huang CY. Proliferation- and migration-enhancing effects of ginseng and ginsenoside Rg1 through IGF-I- and FGF-2-signaling pathways on RSC96 Schwann cells. Cell Biochem. Funct., 27, 186-192 (2009).

24) Liu ZL, Mao JH, Peng AF, Yin QS, Zhou Y, Long XH, Huang SH. Inhibition of fatty acid synthase suppresses osteosarcoma cell invasion and migration via downregulation of the PI3K/Akt signaling pathway in vitro. Mol. Med. Rep., 7, 608-612 (2013).

25) Ould-Yahoui A, Sbai O, Baranger K, Bernard A, Gueye Y, Charrat E, Clement B, Gigmes D, Dive V, Girard SD, Feron F, Khrestchatisky M, Rivera S. Role of matrix metalloproteinases in migration and neurotrophic properties of nasal olfactory stem and ensheathing cells. Cell Transplant., 22, 993-1010 (2013).

26) Gueye Y, Ferhat L, Sbai O, Bianco J, Ould-Yahoui A, Bernard A, Charrat E, Chauvin JP, Risso JJ, Feron F, Rivera S, Khrestchatisky M. Trafficking and secretion of matrix metalloproteinase-2 in olfactory ensheathing glial cells: a role in cell migration? Glia, 59, $750-770$ (2011).

27) Pastrana E, Moreno-Flores MT, Gurzov EN, Avila J, Wandosell F, Diaz-Nido J. Genes associated with adult axon regeneration promoted by olfactory ensheathing cells: a new role for matrix metalloproteinase 2. J. Neurosci., 26, 5347-5359 (2006).

28) Miragall F, Kadmon G, Schachner M. Expression of L1 and N-CAM cell adhesion molecules during development of the mouse olfactory system. Dev. Biol., 135, 272-286 (1989).

29) Witheford M, Westendorf K, Roskams AJ. Olfactory ensheathing cells promote corticospinal axonal outgrowth by a L1 CAM-dependent mechanism. Glia, 61, 1873-1889 (2013).

30) Li X, Yang Z, Song W, Zhou L, Li Q, Tao K, Zhou J, Wang X, Zheng Z, You N, Dou K, Li H. Overexpression of Bmi-1 contributes to the invasion and metastasis of hepatocellular carcinoma by increasing the expression of matrix metalloproteinase (MMP)2, MMP-9 and vascular endothelial growth factor via the PTEN/ PI3K/Akt pathway. Int. J. Oncol., 43, 793-802 (2013).

31) Chang Y, Wu Q, Tian T, Li L, Guo X, Feng Z, Zhou J, Zhang L, Zhou S, Feng G, Han F, Yang J, Huang F. The influence of SRPK1 on glioma apoptosis, metastasis, and angiogenesis through the PI3K/Akt signaling pathway under normoxia. Tumour Biol., 36, 6083-6093 (2015).

32) Zhang Q, Liu G, Wu Y, Sha H, Zhang P, Jia J. BDNF promotes 
EGF-induced proliferation and migration of human fetal neural stem/progenitor cells via the PI3K/Akt pathway. Molecules, 16, 10146-10156 (2011).

33) Xia Z, Zhang N, Ding D. Proliferation and migration of hepatoblastoma cells are mediated by IRS-4 via PI3K/Akt pathways. Int. J. Clin. Exp. Med., 7, 3763-3769 (2014).

34) Zhou R, Xu L, Ye M, Liao M, Du H, Chen H. Formononetin inhibits migration and invasion of MDA-MB-231 and 4T1 breast cancer cells by suppressing MMP-2 and MMP-9 through PI3K/AKT signaling pathways. Horm. Metab. Res., 46, 753-760 (2014).

35) Seo TB, Baek K, Kwon KB, Lee SI, Lim JS, Seol IC, Kim YS,
Seo YB, Namgung U. Shengmai-san-mediated enhancement of regenerative responses of spinal cord axons after injury in rats. $J$. Pharmacol. Sci., 110, 483-492 (2009).

36) Toupalik P, Bouska I, Vojacek T, Jezkova J. Trauma of cervical spinal cord-An immunohistochemical study. Soud. Lek., 51, 6-8 (2006).

37) Darvishi M, Tiraihi T, Mesbah-Namin SA, Delshad A, Taheri T. Decreased GFAP expression and improved functional recovery in contused spinal cord of rats following valproic acid therapy. Neurochem. Res., 39, 2319-2333 (2014). 